\title{
Local Time and Excursions of Reflected Brownian Motion in a Wedge*
}

\author{
Dedicated to Professor $K_{\text {. Itô }}$
}

By

\author{
R. J. WILLIAMS**
}

\begin{abstract}
When the vertex is a regular point for reflected Brownian motion in a wedge, with a constant direction of reflection on each side of the wedge, the law of the excursions from the vertex is determined in the following sense. The nature of the local time at the vertex and the Laplace transform of the entrance law at that point are explicitly given. In particulat, it is shown that the inverse local time at the vertex is a stable subordinator of index $\alpha / 2$ where $0<\alpha \equiv\left(\theta_{1}+\theta_{2}\right) / \xi<2$. Here $\xi$ is the angle of the wedge $(0<\xi<2 \pi)$ and $\theta_{1}, \theta_{2}$ are the angles of reflection on the two sides of the wedge measured from the inward normals, with positive angles being toward the vertex $\left(-\pi / 2<\theta_{1}, \theta_{2}<\pi / 2\right)$. Excursions from the vertex are shown to hit the boundary of the wedge immediately. As a bonus, the invariant measure for the reflected Brownian motion is readily obtained from the Laplace transform of the entrance law, thus verifying an earlier derivation of this result.

AMS 1980 subject classifications. Primary 60J65. Secondary 60J55, 60J60.
\end{abstract}

Keywords and phrases. Brownian motion, oblique reflection, excursions, local time, entrance law.

\section{$\S \mathbb{1}$. Introduction}

Consider a strong Markov process with continuous sample paths that loosely speaking has the following three properties.

(a) The state space is an infinite two-dimensional wedge, and the process behaves in the interior of the wedge like ordinary Brownian motion.

(b) The process reflects instantaneously at the boundary of the wedge, the direction of reflection being constant along each side.

Communicated by S. Matsuura, September 1, 1986.

* This research was conducted while the author was visiting the Institute for Mathematics and its Applications with support provided by the National Science Foundation and the Air Force Office of Scientific Research.

** Department of Mathematics, University of California at San Diego, La Jolla CA 92093, U.S.A. 
(c) The amount of the time that the process spends at the vertex of the wedge has Lebesgue measure zero.

In [16], necessary and sufficient conditions for existence of such a process were given and the process was characterized in law as the unique solution of a submartingale problem. In this paper, when the vertex is a regular point, the nature of the set of times that the process is at the vertex and the excursions of the process away from the vertex between these times is studied. To facilitate the formal description of the process and of the main results obtained here, the following notation is introduced.

The wedge state space is given in polar coordinates by

$$
S=\{(r, \theta): 0 \leqslant \theta \leqslant \xi, r \geqslant 0\}
$$

where $\xi \in(0,2 \pi)$ is the angle of the wedge. The points $(0, \theta): 0 \leqslant \theta \leqslant \xi$ are all identified as the origin $\{0\}$ in $\mathbb{R}^{2}$. The two sides of the wedge are denoted by

$$
\partial S_{1}=\{(r, \theta): \theta=0, r \geqslant 0\} \quad \text { and } \quad \partial S_{2}=\{(r, \theta): \theta=\xi, r \geqslant 0\} .
$$

The directions of reflection on the two sides of the wedge are specified by constant vectors $v_{1}$ and $v_{2}$, normalized such that for $j=1,2, v_{j} \cdot n_{j}=1$ where $n_{j}$ is the unit normal to $\partial S_{j} \backslash\{0\}$ that points into $S$. For each $j$, define the angle of reflection $\theta_{j}$ to be the angle between $n_{j}$ and $v_{j}$ such that $\theta_{j}$ is positive if and only if $v_{j}$ points towards the origin. Note that $-\pi / 2<\theta_{j}<\pi / 2$. Define $\alpha=\left(\theta_{1}+\theta_{2}\right) / \xi$.

Let $C_{S}$ denote the space of continuous functions $w:[0, \infty) \rightarrow S$. For each $t \geqslant 0$, let $\mathscr{M}_{t} \equiv \sigma\{w(s): 0 \leqslant s \leqslant t\}$, the $\sigma$-algebra of subsets of $C_{s}$ generated by the coordinate maps $w \rightarrow w(s)$ for $0 \leqslant s \leqslant t$. Similarly, let $\mathscr{H} \equiv \sigma\{w(s)$ : $0 \leqslant s<\infty\}$. For each non-negative integer $n$ and $F \subset \boldsymbol{R}^{2}$, let $C^{n}(F)$ denote the set of real-valued functions that are $n$-times continuously differentiable in some domain containing $F$. Let $C_{b}^{n}(F)$ denote the set of functions in $C^{n}(F)$ that together with their partial derivatives up to and including those of order $n$ are bounded on $F$. Let $C_{c}^{n}(F)$ denote those functions in $C_{b}^{n}(F)$ whose support is a compact subset of $\boldsymbol{R}^{2}$. If $n=0$, the superscript $n$ will be omitted. Define the differential operators

$$
D_{j}=v_{j} \cdot \nabla \text { for } j=1,2,
$$

and let $\Delta$ be the two-dimensional Laplacian.

A solution of the submartingale problem is a family of probability measures 
$\left\{P^{x}, x \in S\right\}$ on $\left(C_{S}, \mathscr{M}\right)$ such that the following conditions (i)-(iii) hold for each $x \in S$.

(i) $P^{x}(w(0)=x)=1$.

(ii) For each $f \in C_{b}^{2}(S)$,

$$
f(w(t))-\frac{1}{2} \int_{0}^{t} \Delta f(w(s)) d s
$$

is a $P^{x}$-submartingale on $\left(C_{S}, \mathscr{M},\left\{\mathscr{H}_{t}\right\}\right)$ whenever $f$ is constant in a neighborhood of the origin and satisfies

$$
D_{j} f \geqslant 0 \text { on } \partial S_{j} \text { for } j=1,2 \text {. }
$$

(iii) $P^{x}\left[\int_{0}^{\infty} 1_{\{0\}}(w(t)) d t\right]=0$.

Convention. Here $P^{x}$ is used to denote both probability and expectation with respect to $P^{x}$. This convention will be used for all measures and integrals with respect to those measures appearing in this paper.

The following results were proved in [16]. If $\alpha<2$, there is a unique solution $\left\{P^{x}, x \in S\right\}$ of the submartingale problem. If $\alpha \geqslant 2$, there is no solution of the submartingale problem. However, if condition (iii) is removed, then there is a unique solution and the associated probability measures $\left\{P^{x}, x \in S\right\}$ are concentrated on those paths that reach the vertex and then remain there. In either case $(\alpha<2$ or $\alpha \geqslant 2)$, the family $\left\{P^{x}, x \in S\right\}$ has the strong Markov property. Furthermore, for $x \neq 0, w(\cdot)$ reaches the vertex of the wedge with $P^{x}$-probability zero if $\alpha \leqslant 0$ and with $P^{x}$-probability one if $\alpha>0$.

For each $t \geqslant 0$ and $w \in C_{S}$, define

$$
Z(t, w)=w(t) .
$$

Let $\mathcal{B}_{S}$ denote the Borel $\sigma$-algebra on $S$. Let $\mathscr{F}$ denote the usual completion of $\mathscr{M}$, and $\mathscr{F}_{t}$ the usual completion of $\mathscr{M}_{t}$ in $\mathscr{M}$, with respect to $\left\{P^{\mu} \equiv\right.$ $\int_{S} \mu(d x) P^{x}(\cdot): \mu$ is a finite measure on $\left.\left(S, \mathscr{B}_{S}\right)\right\}$. Then [17],

$$
\left(C_{S}, \mathscr{F}, \mathscr{H}_{t}, Z(t), \theta_{t}, P^{x}\right)
$$

is a Hunt process with state space $\left(S, \mathcal{B}_{S}\right)$, where $\theta_{t}$ is the usual shift operator. For brevity, this Hunt process will often be simply denoted by $Z$. Since $\partial S$ is of zero potential for $Z$ [17] and $Z$ behaves like Brownian motion in $S \backslash \partial S$, it follows that Lebesgue measure is a reference measure for $Z$. For more details on Hunt processes and reference measures, see Blumenthal and Gretoor [3]. 
When $\alpha \leqslant 0$, the set of times

$$
\Lambda \equiv\{t \geqslant 0: Z(t)=0\}
$$

for which $Z$ is at the vertex of the wedge is either $P^{x}$-a.s. empty $(x \neq 0)$ or $P^{x}$ a.s. consists of the time $t=0(x=0)$. When $\alpha \geqslant 2$, since $Z$ is absorbed at the vertex in this case, the set $\Lambda$ is $P^{x}$-a.s. a semi-infinite interval $\left[T_{0}, \infty\right)$ where

$$
T_{0}=\inf \{t>0: Z(t)=0\} .
$$

However, for $0<\alpha<2$, it is shown in Section 2 that the (recurrent) vertex is a regular point for itself relative to $Z$. This case will be the focus of attention for the remainder of this paper and so it is assumed henceforth that $0<$ $\alpha<2$. Since the vertex $\{0\}$ is regular for itself, there is a unique (up to a scalar multiple) non-decreasing perfect continuous additive functional whose support is $\{0\}$ [3; Theorem V.3.13, p. 216]. The symbol $L$ will be used to denote this functional normalized to satisfy

$$
P^{0}\left[\int_{0}^{\infty} e^{-t} d L(t)\right]=1
$$

and $L$ will be referred to as the local time of $Z$ at $\{0\}$. Two representations for this local time are given in Section 2. First, for $\phi: S \rightarrow R$ defined by

$$
\phi(r, \theta)=r^{\alpha} \cos \left(\alpha \theta-\theta_{1}\right), r \geqslant 0,0 \leqslant \theta \leqslant \xi,
$$

it is shown that $\phi(Z)$ is a local $P^{x}$-submartingale for each $x \in S$. Then $L$ is a constant $(c>0)$ multiple of the continuous, adapted, non-decreasing process in the Doob-Meyer decomposition of $\phi(Z)$. Secondly, it is shown that for each $x \in S$ and $t \geqslant 0$,

$$
L(t)=c \lim _{\varepsilon \rightarrow 0} \varepsilon^{1-(\alpha / 2)} \int_{0}^{t} 1_{[0, \varepsilon)}(\phi(Z(s))) \psi(Z(s)) d s
$$

where the limit is in probability (with respect to $P^{x}$ ), and

$$
\psi(r, \theta)=\frac{\alpha(2-\alpha)}{2}\left(\cos \left(\alpha \theta-\theta_{1}\right)\right)^{(2 / \alpha)-2}, \quad 0 \leqslant \theta \leqslant \xi, r>0 .
$$

The right continuous inverse $\tau$ of $L$, defined by

$$
\tau(t)=\inf \{s \geqslant 0: L(s)>t\}, t \geqslant 0,
$$

is a strictly increasing subordinator under $P^{0}$. Using a Brownian-like scaling property of $Z$, and the first characterization of $L$, it is proved that $\tau$ is a stable subordinator of index $\alpha / 2$ and rate 1 under $P^{0}$, so that 


$$
P^{0}\left[e^{-\lambda \tau(t)}\right]=e^{-t \kappa(\lambda)}, \lambda>0,
$$

where $\kappa(\lambda)=\lambda^{\alpha / 2}$. It follows [2] that $P^{0}$-a.s., the set of times $A$ that $Z$ is at the vertex has Hausdorff dimension $\alpha / 2$ and its Hausdorff measure function is known [15].

The complement of the closed set $A$ in $\mathbb{R}_{+}=[0, \infty)$ is a countable union of disjoint open intervals and the pieces of the path of $Z$ over these intervals constitute the excursions of $Z$ away from the vertex. The law of these excursions is determined by the entrance law at the vertex together with the transition probabilities for $Z$ killed at the time $T_{0}$. In Section 3, the Laplace transform $\left\{\eta^{\lambda}, \lambda>0\right\}$ of this entrance law is shown to be given by

$$
\eta^{\lambda}(f)=\frac{\chi\left(f-\lambda R_{\partial}^{\lambda} f\right)}{\chi\left(\psi_{1}\right)} \text { for all } f \in C_{c}(S)
$$

where

$$
R_{\partial}^{\lambda} f(x)=\int_{0}^{\infty} e^{-\lambda t} P^{x}\left(f(Z(t)) ; t<T_{0}\right) d t
$$

is the resolvent for $Z$ killed at the time $T_{0}$, and

$$
\begin{gathered}
\psi_{1}(x)=P^{x}\left(e^{-T_{0}}\right)=1-R_{\partial}^{1} 1, \\
\chi(h)=\frac{4 \pi^{2}}{\theta_{1}+\theta_{2}} \int_{0}^{\xi} \int_{0}^{\infty}(h \rho)(r, \theta) r d r d \theta,
\end{gathered}
$$

for

$$
\rho(r, \theta)=r^{-\alpha} \cos \left(\alpha \theta-\theta_{1}\right), r>0,0 \leqslant \theta \leqslant \xi .
$$

The expressions $\chi\left(f-\lambda R_{\partial}^{\lambda} f\right)$ and $\chi\left(\psi_{1}\right)$ in (1.12) are well defined because [16, Lemma 3.2], $R_{\partial}^{\lambda} f(x)$ and $\psi_{1}(x)$ are $O\left(e^{-\beta|x|}\right)$ as $|x| \rightarrow \infty$ for some $\beta>0$. From (1.12) it is readily deduced that $\rho(x) d x$ is an invariant measure for $Z$ [6]. Finally, in Section 3, it is shown that the excursions of $Z$ from the vertex hit the boundary of the wedge immediately. Thus they do not share the local properties of Brownian motion starting from the vertex and conditioned to stay initially in the interior of the wedge.

In recent work, Le Gall [10] has independently obtained the Hausdorff dimension of the set $A$ for reflected Brownian motions in a wedge satisfying $\theta_{1}=\theta_{2}=\xi-\frac{\pi}{2}$ and $\frac{\pi}{2}<\xi<\pi$. To deduce this, Le Gall shows that such a reflected Brownian motion can be represented as the process $\{W(t)-V(t)$, $t \geqslant 0\}$ where $W$ is a two-dimensional Brownian motion and $V(t)$ is the vertex 
of the smallest wedge with angle $\xi$ and fixed orientation that contains the path of $W$ up to time $t$.

\section{§ 2. Local Time at the Vertex}

Recall that in the sequel it is assumed that $0<\alpha<2$. The following Brownian-like scaling property of $Z$ will be used several times in this paper.

Lemma 2.1. Let $x \in S$ and $\lambda>0$. Then for each $A \in \mathscr{F}$,

$$
P^{x}(A)=P^{\lambda x}\left(\lambda^{-1} w\left(\lambda^{2} \cdot\right) \in A\right) .
$$

In particular, for $T_{0}$ defined by (1.5) and $t \geqslant 0$,

$$
P^{x}\left(T_{0}(w) \leqslant t\right)=P^{\lambda x}\left(T_{0}\left(\lambda^{-1} w\left(\lambda^{2} \cdot\right)\right) \leqslant t\right)=P^{\lambda x}\left(T_{0}(w) \leqslant \lambda^{2} t\right) .
$$

Proof. The proof of this lemma is similar to that of Lemma 2.2 in [18], but is included here for completeness. For each $A \in \mathscr{F}$, let $Q^{x}(A)$ denote the right member of (2.1). By the characterization of $P^{x}$, to prove (2.1), it suffices to verify that $Q^{x}$ satisfies properties (i)-(iii) of the submartingale problem, with $Q^{x}$ in place of $P^{x}$.

Properties (i) and (iii) follow immediately from those for $P^{\lambda x}$. For property (ii), suppose $f \in C_{b}^{2}(S)$ is constant in a neighborhood of the origin and satisfies (1.2). Then $f\left(\lambda^{-1} \cdot\right)$ also satisfies (1.2) and so by applying the submartingale property of $P^{\lambda x}$ to this function, and performing a change of variable in the time integration (from $s$ to $\lambda^{-2} s$ ), we conclude that

$$
\left\{f\left(\lambda^{-1} w\left(\lambda^{2} t\right)\right)-\frac{1}{2} \int_{0}^{t}(\Delta f)\left(\lambda^{-1} w\left(\lambda^{2} s\right)\right) d s, \mathscr{M}_{\lambda^{2}} t, t \geqslant 0\right\}
$$

is a $P^{\lambda x}$-submartingale. By the definition of $Q^{x}$, this implies (1.1) is a $Q^{x}$ submartingale.

The result for $T_{0}$ follows immediately from (2.1).

As an easy consequence of Lemma 2.1, we now conclude that the vertex is a regular point for $Z$.

\section{Lemma 2.2.}

$$
P^{0}\left(T_{0}=0\right)=1 .
$$

Proof. By setting $x=0$ in (2.2) and letting $\lambda \rightarrow \infty$ and then $t \downarrow 0$, (2.3) follows from the fact that $P^{0}\left(T_{0}(w)<\infty\right)=1$.

In the sequel, a generalization of the submartingale property of $P^{x}$ will 
be needed. A version of this was given as Theorem 3.5 in [16]. The following local submartingale version of that theorem can easily be obtained by standard approximation and stopping arguments.

Lemma 2.3. Suppose $h \in C_{b}(S \backslash\{0\})$ and $f \in C(S) \cap C^{2}(S \backslash\{0\})$ such that

$$
\begin{gathered}
\frac{1}{2} \Delta f=h \quad \text { in } S \backslash\{0\} \\
D_{j} f \geqslant 0 \text { on } \partial S_{j} \backslash\{0\} \text { for } j=1,2, \\
|f(z)-f(0)|=o(\phi(z)) \text { as }|z| \rightarrow 0 .
\end{gathered}
$$

Then for each $x \in S$,

$$
f(Z(t))-\int_{0}^{t}\left(1_{s \backslash\{0\}} h\right)(Z(s)) d s
$$

is a local submartingale on $\left(C_{S}, \mathscr{F},\left\{\mathscr{F}_{t}\right\}, P^{x}\right)$.

Lemma 2.4. For each $x \in S, \phi(Z)$ is a local submartingale on $\left(C_{S}, \mathscr{F}_{1},\left\{\mathscr{F}_{t}\right\}\right.$, $\left.P^{x}\right)$.

Proof. Let $\beta=\left(\hat{\theta}_{1}+\hat{\theta}_{2}\right) / \xi$ where $\hat{\theta}_{1}$ and $\hat{\theta}_{2}$ are angles in $\left(-\frac{\pi}{2}, \frac{\pi}{2}\right)$ such that $\hat{\theta}_{1}>\theta_{1}$ and $\hat{\theta}_{2}>\theta_{2}$. Then $\beta>\alpha$ and

$$
\phi^{\beta}(r, \theta) \equiv r^{\beta} \cos \left(\beta \theta-\hat{\theta}_{1}\right), r \geqslant 0,0 \leqslant \theta \leqslant \xi
$$

is harmonic in $S \backslash\{0\}$,

$$
D_{j} \phi^{\beta}=\beta r^{\beta-1} \sin \left(\hat{\theta}_{j}-\theta_{j}\right) / \cos \theta_{j} \geqslant 0 \text { on } \partial S_{j} \backslash\{0\} \text { for } j=1,2 \text {, }
$$

and $\phi^{\beta}(z)=o(\phi(z))$ as $|z| \rightarrow 0$. It then follows from Lemma 2.3 that $\phi^{\beta}(Z(\cdot))$ is a local $P^{x}$-submartingale for each $x \in S$. Thus, for $x \in S, R>0$ and $\sigma_{R} \equiv$ inf $\{t \geqslant 0: \phi(Z(t)) \geqslant R\}, \phi^{\beta}\left(Z\left(\cdot \wedge \sigma_{R}\right)\right)$ is a bounded $P^{x}$-submartingale, where the bound is uniform for all $\beta \in(\alpha, \pi / \xi)$. As $\beta \downarrow \alpha, \phi^{\beta}\left(Z\left(\bullet \wedge \sigma_{R}\right)\right)$ converges pointwise to $\phi\left(Z\left(\cdot \wedge \sigma_{R}\right)\right)$ and so it follows from bounded convergence for conditional expectations that $\phi\left(Z\left(\bullet \wedge \sigma_{R}\right)\right)$ is also a $P^{x}$-submartingale. Hence, since $R>0$ was arbitrary, $\phi(Z)$ is a local $P^{x}$-submartingale.

In the following, adapted means adapted to $\left\{\mathscr{F}_{t}\right\}$.

For each $x \in S$, since $\phi(Z)$ is a local submartingale on $\left(C_{S}, \mathscr{F},\left\{\mathscr{F}_{t}\right\}, P^{x}\right)$, it can be uniquely decomposed:

$$
\phi(Z(t))-\phi(Z(0))=M(t)+A(t), t \geqslant 0
$$

where $M$ is a continuous local martingale, $A$ is a continuous, adapted, nondecreasing process and $M(0)=A(0)=0 P^{x}$-a.s. A priori, the processes $M$ and 
$A$ may depend upon $x$. However, Cinlar, Jacod, Protter and Sharpe [4, Theorem 3.12] have shown that one may choose continuous, adapted processes $M$ and $A$ (not depending on $x$ ) such that the above holds for all $x$. Moreover, since $\phi(Z(\cdot))-\phi(Z(0))$ is an additive process, $M$ and $A$ may also be chosen to be additive [4, Theorem 3.18]. It will be assumed in the sequel that $M$ and $A$ are chosen in this way. In the next lemma it is shown that the support of $A$ is $\{0\}$, or in other words, " $A$ increases only when $Z$ is at the origin".

Lemma 2.5. Let $x \in S$. Then

$$
\int_{0}^{\infty} 1_{s \backslash(0\}}(Z(s)) d A(s)=0 \quad P^{x} \text {-a.s. }
$$

Proof. It suffices to prove that for each $\varepsilon>0, P^{x}$-a.s.:

$$
A^{\mathrm{q}}(t) \equiv \int_{0}^{t} 1_{[\varepsilon, \infty)}(\phi(Z(s))) d A(s)=0 \text { for all } t \geqslant 0,
$$

or equivalently,

$$
\tau_{\mathrm{\varepsilon}} \equiv \inf \left\{t \geqslant 0: A^{\mathrm{\varepsilon}}(t)>0\right\}
$$

is $P^{x}$-a.s. infinite. On $\left\{\tau_{\varepsilon}<\infty\right\}, \phi\left(Z\left(\tau_{\varepsilon}\right)\right) \geqslant \varepsilon$ and

$$
A(t)-A\left(\tau_{\varepsilon}\right)>0 \text { for } \tau_{\varepsilon}<t<T_{0}^{\varepsilon}
$$

where

$$
T_{0}^{\varepsilon} \equiv \inf \left\{t \geqslant \tau_{\mathrm{\varepsilon}}: Z(t)=0\right\}>\tau_{\mathrm{g}} .
$$

On the other hand, since $\phi$ is harmonic in $S \backslash\{0\}$ and $D_{j} \phi=0$ on $\partial S_{j} \backslash\{0\}, j=$ 1,2 , it follows from a local version of the submartingale property of $P^{x}$ that

$$
\left\{\left(\phi\left(Z\left(t \wedge T_{0}^{\varepsilon}\right)\right)-\phi\left(Z\left(t \wedge \tau_{\varepsilon}\right)\right)\right) 1_{\left\{\tau_{\varepsilon}<\infty\right\}}, t \geqslant 0\right\}
$$

defines a local $P^{x}$-martingale. But this contradicts $(2.8)$, unless $P^{x}\left(\tau_{\varepsilon}<\infty\right)$ $=0$.

Theorem 2.6. Let $L$ denote the local time of $Z$ at $\{0\}$, as defined in Section 1 . Then there is a constant $c \in(0, \infty)$ such that for each $x \in S, P^{x}$-a.s.,

$$
L(t)=c A(t), t \geqslant 0 .
$$

Moreover, for each $\lambda>0$, under $P^{0}$,

$$
\lambda^{-(\alpha / 2)} L(\lambda \cdot \stackrel{d}{=} L(\cdot)
$$


where $\stackrel{d}{=}$ denotes equality in distribution.

Proof. Since $A$ is a continuous additive functional of $Z$, and by Lemma 2.5 the support of $A$ is $\{0\}$, it follows from the definition [3, p. 216] of $L$ that (2.9) holds $P^{x}$-a.s. for each $x \in S$.

For the scaling property of $L$, recall from Lemma 2.1 that under $P^{0}$,

$$
\lambda^{-1 / 2} Z(\lambda \cdot) \stackrel{d}{=} Z(\cdot) \text {. }
$$

Hence, $P^{0}$-a.s. :

$$
\begin{aligned}
\phi(Z(\cdot)) & \stackrel{d}{=} \phi\left(\lambda^{-1 / 2} Z(\lambda \cdot)\right)=\lambda^{-(\alpha / 2)} \phi(Z(\lambda \circ)) \\
& =\lambda^{-\alpha / 2}(M(\lambda \cdot)+A(\lambda \cdot)) .
\end{aligned}
$$

It follows from the uniqueness of the decomposition of $\phi(Z(\cdot))$ that $P^{0}$-a.s.,

$$
A(\cdot) \stackrel{d}{=} \lambda^{-\infty / 2} A(\lambda \cdot) \text {. }
$$

This, together with (2.9), yields (2.10).

Corollary 2.7. Let $\tau$ be the right continuous inverse of $L$, defined by (1.10). Then under $P^{0}, \tau$ is a strictly increasing stable subordinator of index $\alpha / 2$ and rate 1, i.e., (1.11) holds.

Proof. It is well known that $\tau$ is a strictly increasing subordinator [3, pp. 217-219]. Now, for $t \geqslant 0$, under $P^{0}$,

$$
\begin{aligned}
\lambda^{-1} \tau\left(\lambda^{\alpha / 2} t\right) & =\inf \left\{\lambda^{-1} s \geqslant 0: L(s)>\lambda^{\alpha / 2} t\right\} \\
& =\inf \left\{s \geqslant 0: \lambda^{-\alpha / 2} L(\lambda s)>t\right\} \\
& \stackrel{d}{=} \inf \{s \geqslant 0: L(s)>t\}
\end{aligned}
$$

where (2.10) has been used in the last line above. Hence $\tau$ is a stable subordinator of index $\alpha / 2[3,14]$. The fact that $\tau$ has rate 1 comes from the normalization (1.6) of $L$ :

$$
P^{0}\left[\int_{0}^{\infty} e^{-\tau(t)} d t\right]=P^{0}\left[\int_{0}^{\infty} e^{-t} d L(t)\right]=1
$$

In the next lemma, an alternative representation is given for $A$, hence for $L$.

Lemma 2.8. Let $x \in S$. Then for each $t \geqslant 0$,

$$
A(t)=\lim _{\varepsilon \rightarrow 0} \varepsilon^{1-(\alpha / 2)} \int_{0}^{t} 1_{[0, \varepsilon)}(\phi(Z(s))) \psi(Z(s)) d s
$$


where the limit is in probability (with respect to $P^{x}$ ) and $\psi$ is defined by (1.9).

Remark. Since $\phi(Z)$ is only known to be a local submartingale, a stronger form of convergence in (2.11), such as convergence in $L^{2}\left(C_{S}, \mathscr{F}, P^{x}\right)$ as $\varepsilon \rightarrow 0$, was not obtained here. As pointed out by J.F. Le Gall (private communication), similar results to (2.11) can be obtained by applying knowledge of the recurrence and invariant measure of $Z$ [17], together with ergodic theorems for additive functionals. One advantage of the computational proof given here is that a precise representation (without undetermined constants) is obtained for $A(t)$.

Proof. First, observe that since $Z$ spends zero time at the vertex, in the sense of Lebesgue measure, we may replace $1_{[0, \varepsilon)}$ by $1_{(0, \varepsilon)}$ in $(2.11)$. The proof of (2.11) is similar in spirit to that for the representation of the local time of a one-dimensional reflected Brownian motion. In particular, we need to apply the submartingale property of $P^{x}$ to a function whose Laplacian is of the order of $1_{(0, \varepsilon)}(\phi(z))$. Since $\phi$ satisfies the boundary condition $D_{j} \phi=0$ on $\partial S_{j} \backslash\{0\}$ for $j=1,2$, so will a function of the form $f_{\varepsilon}(z)=g_{\varepsilon}(\phi(z))$, where $g_{\varepsilon}$ is a differentiable function of a real variable. Moreover, the function $g_{\varepsilon}$ can be chosen so that the Laplacian of $f_{\mathrm{q}}$ has the aforementioned property (see (2.14) below). The following functions $g_{\varepsilon}$ were found in this way.

For each $\varepsilon>0$, let $g_{\varepsilon}:[0, \infty) \rightarrow[0, \infty)$ be defined by

$$
g_{\varepsilon}(y)\left\{\begin{array}{l}
\frac{\alpha}{2} y^{2 / \alpha} \text { for } 0 \leqslant y<\varepsilon \\
\left((y-\varepsilon)+\frac{\alpha \varepsilon}{2}\right) \varepsilon^{(2 / \alpha)-1} \text { for } \varepsilon \leqslant y<\infty .
\end{array}\right.
$$

Then $g_{\varepsilon}$ is once continuously differentiable on $[0, \infty)$ (differentiability being from the right at 0$)$ and twice continuously differentiable on $(0, \infty) \backslash\{\varepsilon\}$. Indeed, since $2 / \alpha>1$, we have

$$
g_{\varepsilon}^{\prime}(y)= \begin{cases}y^{(2 / \alpha)-1} & \text { for } 0 \leqslant y<\varepsilon \\ \varepsilon^{(2 / \alpha)-1} & \text { for } \varepsilon \leqslant y<\infty\end{cases}
$$

and

$$
g_{\varepsilon}^{\prime \prime}(y)= \begin{cases}\left(\frac{2}{\alpha}-1\right) y^{(2 / \alpha)-2} & \text { for } 0<y<\varepsilon, \\ 0 & \text { for } \varepsilon<y<\infty\end{cases}
$$

Then $f_{\mathrm{\varepsilon}}(z) \equiv g_{\mathrm{e}}(\phi(z))$ satisfies [16, Theorem 2.3]: 


$$
D_{j} f_{\varepsilon}(z)=\left(v_{j} \circ \nabla \phi(z)\right) g_{\varepsilon}^{\prime}(\phi(z))=0, z \in \partial S_{j} \backslash\{0\},
$$

and on $\{z \in S: \phi(z) \neq 0$ or $\varepsilon\}$,

$$
\begin{aligned}
\Delta f_{\varepsilon}(z) & =g_{\varepsilon}^{\prime \prime}(\phi(z))|\nabla \phi(z)|^{2}+g_{\varepsilon}^{\prime}(\phi(z)) \Delta \phi(z) \\
& =2 \psi(z) 1_{(0, \varepsilon)}(\phi(z))
\end{aligned}
$$

since on $S \backslash\{0\}, \phi$ is harmonic and satisfies

$$
|\nabla \phi(z)|^{2}=\alpha^{2} r^{2 \alpha-2}=\frac{2 \alpha}{2-\alpha} \psi(z)(\phi(z))^{2-(2 / \alpha)} .
$$

Note also that since $\frac{2}{\alpha}>1$,

$$
f_{\varepsilon}(z)=o(\phi(z)) \text { as }|z| \rightarrow 0 .
$$

Thus, $f_{\mathrm{z}}$ and $-f_{\mathrm{z}}$ satisfy all of the hypotheses of Lemma 2.3 , except that $f_{\mathrm{\varepsilon}}$ is not twice continuously differentiable across $\{z \in S: \phi(z)=\varepsilon\}$. However, since $Z$ spends zero time on this set (in the sense of Lebesgue measure), we can apply Lemma 2.3 to suitable approximations to $f_{\varepsilon}$ and then pass to the limit to conclude that this lemma also applies to $f_{\varepsilon}$ and $-f_{\varepsilon}$ (cf. [17, p. 776]). Hence,

$$
g_{\mathrm{e}}(\phi(Z(t)))-\int_{0}^{t} 1_{(0, \mathrm{e})}(\phi(Z(s))) \psi(Z(s)) d s
$$

is a local martingale on $\left(C_{S}, \mathscr{F},\left\{\mathscr{F}_{t}\right\}, P^{x}\right)$.

On the other hand, since $\phi(Z)$ is a local submartingale under $P^{x}$ and $g_{\varepsilon}$ is a convex function, we can apply the generalized Itô formula [8, pp. 186188] to conclude that $P^{x}$-a.s. for all $t \geqslant 0$,

$$
\begin{aligned}
& g_{\varepsilon}(\phi(Z(t)))-g_{\varepsilon}(\phi(Z(0))) \\
& \quad=\int_{0}^{t} g_{\varepsilon}^{\prime}(\phi(Z(s))) d \phi(Z(s))+\frac{1}{2} \int_{0}^{t}\left(1_{(0, \varepsilon)} g_{\varepsilon}^{\prime \prime}\right)(\phi(Z(s))) d[M](s),
\end{aligned}
$$

where $[M]$ denotes the quadratic variation of the local martingale part $M$ of $\phi(Z)$. Since $g_{\varepsilon}^{\prime}(0)=0$ and $A$ increases only when $\phi(Z)$ is zero, it follows that the first integral in (2.18) is really the local martingale

$$
\int_{0}^{t} g_{\varepsilon}^{\prime}(\phi(Z(s))) d M(s)
$$

Then, comparing (2.17) with (2.18) and invoking uniqueness of the DoobMeyer decomposition of the continuous local submartingale $g_{\varepsilon}(\phi(Z))$, we obtain $P^{x}$-a.s. : 


$$
\begin{aligned}
& \frac{1}{2} \int_{0}^{t}\left(1_{(0, \varepsilon)} g_{\varepsilon}^{\prime \prime}\right)(\phi(Z(s))) d[M](s) \\
& \quad=\int_{0}^{t} 1_{(0, \mathrm{\varepsilon})}(\phi(Z(s))) \psi(Z(s)) d s, t \geqslant 0 .
\end{aligned}
$$

Thus, (2.18) is equivalent to

$$
\begin{aligned}
& g_{\mathrm{e}}(\phi(Z(t)))-g_{\mathrm{\varepsilon}}(\phi(Z(0))) \\
& \quad=\int_{0}^{t} g_{\varepsilon}^{\prime}(\phi(Z(s))) d M(s)+\int_{0}^{t} 1_{(0, \mathrm{\varepsilon})}(\phi(Z(s))) \psi(Z(s)) d s .
\end{aligned}
$$

The next step is to multiply (2.21) by $\varepsilon^{1-(2 / \alpha)}$ and let $\varepsilon \rightarrow 0$. For this, we need to localize $M, A$ and $[M]$. So let $\left\{\tau_{n}\right\}$ be a sequence of stopping times (relative to $\left\{\mathcal{F}_{t}\right\}$ ) tending $P^{x}$-a.s. to infinity such that for each $n \geqslant \phi(x), M^{n}(\cdot)$ $\equiv M\left(\cdot \wedge \tau_{n}\right), A^{n}(\cdot) \equiv A\left(\cdot \wedge \tau_{n}\right)$ and $\left[M^{n}\right](\cdot) \equiv[M]\left(\cdot \wedge \tau_{n}\right)$ are all $P^{x}$-a.s. bounded by $n$. Now, for $n \geqslant \phi(x)$,

$$
\begin{aligned}
& \varepsilon^{1-(2 / \infty)} \int_{0}^{t \wedge \tau_{n}} g_{\varepsilon}^{\prime}(\phi(Z(s))) d M(s) \\
& =\int_{0}^{t}\left(\frac{\phi}{\varepsilon}\right)(2 / \infty)-1(Z(s)) 1_{(0, \varepsilon)}(\phi(Z(s))) d M^{n}(s) \\
& \quad+\int_{0}^{t} 1_{[\varepsilon, \infty)}(\phi(Z(s))) d M^{n}(s) .
\end{aligned}
$$

By the $L^{2}$-isometry for stochastic integrals and since $(\phi / \varepsilon)^{(2 / \alpha)-1} \leqslant 1$ on $\{z \in S$ : $0<\phi(z)<\varepsilon\}$, we have

$$
\begin{aligned}
P^{x} & {\left[\left(\int_{0}^{t}\left(\frac{\phi}{\varepsilon}\right)^{(2 / \alpha)-1}(Z(s)) 1_{(0, \varepsilon)}(\phi(Z(s))) d M^{n}(s)\right)^{2}\right] } \\
& \leqslant P^{x}\left[\int_{0}^{t} 1_{(0, \varepsilon)}(\phi(Z(s))) d\left[M^{n}\right](s)\right]
\end{aligned}
$$

where the right member above tends to zero as $\varepsilon \rightarrow 0$ by dominated convergence. Thus, for each $t \geqslant 0$, the middle integral in (2.22) tends to zero in $L^{2}\left(C_{S}, \mathscr{F}, P^{x}\right)$ as $\varepsilon \rightarrow 0$. Similarly, the last integral in (2.22) is Cauchy and hence converges in $L^{2}\left(C_{S}, \mathscr{F}, P^{x}\right)$ as $\varepsilon \rightarrow 0$. The limit is the stochastic integral

$$
\int_{0}^{t} 1_{(0, \infty)}(\phi(Z(s))) d M^{n}(s)=\int_{0}^{t \wedge^{\tau n}} 1_{(0, \infty)}(\phi(Z(s))) d M(s) .
$$

Now, as $\varepsilon \rightarrow 0, \varepsilon^{1-(2 / \alpha)} g_{\varepsilon}(\phi(z))$ converges pointwise to $\phi(z)$. Moreover, $\varepsilon^{1-(2 / \alpha)} g_{\varepsilon}\left(\phi\left(Z\left(\cdot \wedge \tau_{n}\right)\right)\right)$ is $P^{x}$-a.s. bounded, uniformly in $\varepsilon$, for each $n \geqslant \phi(x)$. Thus, multiplying (2.21) by $\varepsilon^{1-(2 / \infty)}$ and taking the $L^{2}$-limit as $\varepsilon \rightarrow 0$ with $t \wedge \tau_{n}$ in place of $t$ there, we obtain $P^{x}$-a.s. for each $t \geqslant 0$ and $n \geqslant \phi(x)$, 


$$
\begin{aligned}
\phi\left(Z\left(t \wedge \tau_{n}\right)\right)-\phi(Z(0))= & \int_{0}^{t \wedge \tau_{n}} 1_{(0, \infty)}(\phi(Z(s))) d M(s) \\
& +\lim _{\varepsilon \rightarrow 0} \varepsilon^{1-(2 / \infty)} \int_{0}^{t \wedge \tau_{n}} 1_{(0, \varepsilon)}(\phi(Z(s))) \psi(Z(s)) d s
\end{aligned}
$$

where the limit as $\varepsilon \rightarrow 0$ is in $L^{2}\left(C_{S}, \mathscr{F}, P^{x}\right)$.

But by the uniqueness of the Doob-Meyer decomposition of $\phi\left(Z\left(\circ \wedge \tau_{n}\right)\right)$ $\phi(Z(0))$, we have $P^{x}$-a.s. for each $t \geqslant 0$ and $n \geqslant \phi(x)$,

$$
\begin{gathered}
M\left(t \wedge \tau_{n}\right)=\int_{0}^{t \wedge \tau_{n}} 1_{(0, \infty)}(\phi(Z(s))) d M(s), \\
A\left(t \wedge \tau_{n}\right)=\lim _{\varepsilon \rightarrow 0} \varepsilon^{1-\left(2 / \alpha^{\alpha}\right)} \int_{0}^{t \wedge \tau_{n}} 1_{(0, \varepsilon)}(\phi(Z(s))) \psi(Z(s)) d s,
\end{gathered}
$$

where the limit is in $L^{2}\left(C_{S}, \mathscr{F}, P^{x}\right)$. Since $L^{2}$ convergence implies convergence in probability and $\tau_{n} \rightarrow \infty P^{x}$-a.s. as $n \rightarrow \infty$, it follows from (2.25) that (2.11) holds with the limit as $\varepsilon \rightarrow 0$ in probability relative to $P^{x}$.

\section{§3. Excursions from the Vertex}

First, the notions of excursion space and Poisson point process in the context of this paper are reviewed. For further details, the reader is referred to Itô [7] and Salisbury [12], where excursions of a strong Markov process from a regular point are considered. Further properties and the general theory of excursions from more than a single point are discussed in Getoor [6] and Maisonneuve [11], for example.

Let $(I, \mathscr{Q})$ denote the measurable space of $\left(C_{S}, \mathscr{M}\right)$-valued point functions. That is, adjoin a point $\partial$ to $C_{S}$ and let $\Pi$ be the set of functions $p$ : $(0, \infty) \rightarrow C_{S} \cup\{\partial\}$ such that $p(t)=\partial$ except for countably many $t$. The $\sigma$-algebra $\mathscr{P}$ is generated by the functions $p \rightarrow N(A, p)$ where $N(A, p)$ is the number of times $t$ such that $(t, p(t)) \in A$ where $A \in \mathscr{B} \times \mathscr{M}$ and $\mathscr{B}$ is the $\sigma$-algəbra of Borel subsets of $(0, \infty)$. For $A \in \mathscr{B} \times \mathscr{M}$, define the restriction of $p \in \Pi$ to $A$ by

$$
\left.p\right|_{A}(t)=\left\{\begin{array}{l}
p(t) \text { if }(t, p(t)) \in A \\
\partial \text { otherwise. }
\end{array}\right.
$$

Special cases of this are the killing operators:

$$
k_{t}(p)=\left.p\right|_{(0, t] \times c_{s}} t \geqslant 0 .
$$

The shift operators on $\Pi$ are defined by

$$
\theta_{t}(p)=p(\cdot+t), t \geqslant 0
$$

A Poisson point process on $\left(C_{S}, \mathscr{F}, P^{0}\right)$ with values in $\left(C_{S}, \mathscr{M}\right)$ is a measurable 
function $Y:\left(C_{S}, \mathscr{I}\right) \rightarrow(I, \mathscr{Q})$ such that

(a) $k_{t}(Y)$ is independent of $\theta_{t}(Y)$ for all $t \geqslant 0$,

(b) $\theta_{t}(Y)$ has the same probability law as $Y$ for every $t \geqslant 0$,

(c) for each $t \geqslant 0$, there exists a sequence $\left\{U_{n}=U_{n}(t)\right\}$ of sets in $\mathscr{M}$ such that $U_{n} \uparrow C_{S}$ and for each $n$,

$$
N\left((0, t) \times U_{n}, Y\right)<\infty P^{0} \text {-a.s. }
$$

Under conditions (a)-(c), there is a unique $\sigma$-finite measure $\eta$ on $\mathscr{M}$ such that

$$
P^{0}[N((0, t) \times B)]=t \cdot \eta(B) \text { for each } B \in \mathscr{M} \text { and } t \geqslant 0 .
$$

This measure, called the characteristic measure of $Y$, determines the law of the Poisson point process $Y$.

The excursion process $Y$ of $Z$ with respect to $P^{0}$ is defined as follows. (For aesthetic reasons, the same symbol $Y$ is used here as above, for it will turn out that $Y$ is a Poisson point process.) Recall that $\tau$ is the right continuous inverse of the local time $L$ of $Z$ at $\{0\}$. Define $\tau(0-)=0$ and for each $t>0, s \geqslant 0$, let

$$
Y_{t}(s)=\left\{\begin{array}{cl}
Z(\tau(t-)+s) & \text { if } 0 \leqslant s<\tau(t)-\tau(t-) \\
0 & \text { if } s \geqslant \tau(t)-\tau(t-)>0 \\
0 & \text { if } \tau(t)-\tau(t-)=0 .
\end{array}\right.
$$

In [7], Itô proved that $Y$ is a Poisson point process on $\left(C_{S}, \mathscr{F}, P^{0}\right)$ and the characteristic measure $\eta$ of $Y$ is concentrated on

$$
\left\{w \in C_{S}: 0<T_{0}<\infty, w(t)=0 \text { for } t \geqslant T_{0}\right\}
$$

where $T_{0}=T_{0}(w)$ is the lifetime of the excursion $w$ in $S \backslash\{0\}$, defined by

$$
T_{0}=\inf \{t>0: w(t)=0\} .
$$

Although $\eta$ is a $\sigma$-finite measure, in the sequel, the expression $\eta$-a.s. will be used instead of $\eta$-a.e., because of its intuitive value.

Let $\left\{P_{t}^{a}, t \geqslant 0\right\}$ denote the semigroup of the process $Z$ killed at the time $T_{0}$, i.e., for each $A \in \mathscr{B}_{S}, x \in S$ and $t \geqslant 0$ :

$$
P_{t}^{\mathrm{\partial}}(x, A)=P^{x}\left(Z(t) \in A, t<T_{0}\right) .
$$

For each $t>0$, define a measure $\eta_{t}$ on $\left(S, \mathcal{B}_{S}\right)$ by

$$
\eta_{t}(A)=\eta\left(w \in C_{S}: w(t) \in A, t<T_{0}\right), A \in \mathscr{B}_{S} .
$$

Each $\eta_{t}$ is a finite measure on $S$ and $\left\{\eta_{t}, t>0\right\}$ is an entrance law for the killed 
semigroup $\left\{P_{t}^{\partial}, t \geqslant 0\right\}[6$, p. 247], that is,

$$
\eta_{t+s}=\eta_{t} P_{s}^{\partial} \text { for all } t>0 \text { and } s \geqslant 0 .
$$

Conversely, this entrance law together with the killed semigroup determines the finite-dimensional distributions of $\eta$ (and hence determines $\eta$ ) as follows. For $0<t_{1}<t_{2}<\cdots<t_{n}<\infty$ and $x_{1}, \cdots, x_{n} \in S \backslash\{0\}$,

$$
\begin{aligned}
& \eta\left(w\left(t_{1}\right) \in d x_{1}, \cdots, w\left(t_{n}\right) \in d x_{n}, t_{n}<T_{0}\right) \\
& \quad=\eta_{t_{1}}\left(d x_{1}\right) P_{t_{2}-t_{1}}^{\partial}\left(x_{1}, d x_{2}\right) \cdots P_{t_{n}-t_{n-1}}^{\mathrm{a}}\left(x_{n-1}, d x_{n}\right) .
\end{aligned}
$$

Thus, viewing the killed semigroup as given, to determine the excursion law $\eta$, it suffices to find its entrance law or equivalently the Laplace transform in $t$ of this entrance law:

$$
\eta^{\lambda}(A)=\int_{0}^{\infty} e^{-\lambda l} \eta_{t}(A) d t, \lambda>0, A \in \mathscr{B}_{S} .
$$

The latter is explicitly given in the following theorem.

Theorem 3.1. For each $f \in C_{c}(S), \eta^{\lambda}(f) \equiv \int_{S} f(x) \eta^{\lambda}(d x)$ is given by (1.12).

Proof. The Laplace transform $\eta^{\lambda}$ is related to the resolvent $R^{\lambda}$ of $Z$ by $[6 ;(7.8),(7.20)]$ :

$$
\eta^{\lambda}(f)=\kappa(\lambda) R^{\lambda} f(0), f \in C_{c}(S)
$$

wherc

$$
\kappa(\lambda)=\lambda^{\alpha / 2}
$$

is the characteristic exponent (see (1.11)) for the stable subordinator $\tau$, and

$$
R^{\lambda} f(x)=P^{x}\left[\int_{0}^{\infty} e^{-\lambda t} f(Z(t)) d t\right], \quad x \in S .
$$

From the proof of Theorem 3.10 in [16] we have

$$
R^{\lambda} f(0)=\frac{\chi\left(f-\lambda R_{\partial}^{\lambda} f\right)}{\lambda \chi\left(1-\lambda R_{\partial}^{\lambda} 1\right)}, \quad f \in C_{c}(S)
$$

where $\chi$ is defined by (1.15). In fact, in [16], (3.9) was proved for $f \in C_{c}^{\infty}(S)$ that vanish in a neighborhood of the origin, but the extension to $f \in C_{c}(S)$ is easily obtained. From the scaling property (see Lemma 2.1) of $P^{x}$, it follows that

$$
1-\lambda\left(R_{\partial}^{\lambda} 1\right)(x)=P^{x}\left[e^{-\lambda T_{0}}\right]=P^{2} \bar{\lambda}^{x}\left[e^{-T_{0}}\right]=\psi_{1}(\sqrt{\lambda} x)
$$


where $\psi_{1}$ is defined by (1.14). By combining this with the scaling property of $\rho$ (the density appearing in the definition (1.15) of $\chi$ ), we obtain

$$
\lambda \chi\left(1-\lambda R_{\partial}^{\lambda} 1\right)=\lambda^{\alpha / 2} \chi\left(\psi_{1}\right) .
$$

Substituting this in (3.9) and that in turn in (3.8) yields the desired formula (1.12) for $\eta^{\lambda}(f)$.

Corollary 3.2. The measure with density o relative to Lebesgue measure on $S$ is an invariant measure for $Z$.

Remark. This is consistent with results obtained in [17] where it was shown that, up to a scalar multiple, $\rho(x) d x$ is the unique invariant for $Z$ when $0 \leqslant \alpha<2$.

Proof. By the definition (1.15) of $\chi$ and Theorem 8.1 of Getoor [6], it suffices to prove for each $f \in C_{c}(S), f \geqslant 0$, that

$$
\lim _{\lambda \downarrow 0} \eta^{\lambda}(f)=\frac{\chi(f)}{\chi\left(\psi_{1}\right)}
$$

That is, by (1.12), we must prove that

$$
\lim _{\lambda \downarrow 0} \chi\left(\lambda R_{\partial}^{\lambda} f\right)=0 \text {. }
$$

Fix $f \in C_{c}(S)$. Let $\|f\|=\max _{x \in S}|f(x)|$ and let $R>0$ such that the support of $f$ in $S$ is contained in $\{x \in S: 0 \leqslant \phi(x) \leqslant R\}$ where $\phi$ is defined by (1.7). Then, for each $x \in S$,

$$
\begin{aligned}
\left|\lambda R_{\partial}^{\lambda} f(x)\right| & \leqslant\|f\| \lambda P^{x}\left[\int_{0}^{T_{0}} e^{-\lambda t} 1_{(0, R)}(\phi(Z(t))) d t\right] \\
& =\|f\| P^{\sqrt{\lambda} x}\left[\int_{0}^{T_{0}} e^{-t} 1_{\left(0, R \lambda^{\alpha / 2}\right)}(\phi(Z(t))) d t\right]
\end{aligned}
$$

where the scaling properties of $P^{x}$ and $\phi$ have been used to obtain the last line above. By letting $K \rightarrow \infty$ in Corollary 2.2 of [16], we obtain for each $x \in S$ and $r>0$,

$$
P^{x}\left[\int_{0}^{T_{0}} 1_{(0, r)}(\phi(Z(t))) d t\right] \leqslant a r^{2 / \alpha}
$$

where $a$ is a constant not depending on $r$ or $x$. For each $r>0$, let

$$
T_{r}=\inf \{t \geqslant 0: \phi(Z(t)) \leqslant r\} \text {. }
$$

Then it follows from the proof of Lemma 3.2 in [16] that there are constants $\beta>0$ and $K>0$ such that for all sufficiently small $r$, 


$$
P^{x}\left[e^{-T} r\right] \leqslant K e^{-\beta|x|}, x \in S .
$$

By (3.12) and the scaling of $\rho$, we have

$$
\begin{aligned}
& \left|\int_{S} \lambda R_{\partial}^{\lambda} f(x) \rho(x) d x\right| \\
& \leqslant\|f\| \lambda^{(\alpha / 2)-1} \int_{S} P^{x}\left[\int_{0}^{T_{0}} e^{-t} 1_{\left(0, R \lambda^{\alpha / 2}\right)}(\phi(Z(t))) d \iota\right] \rho(x) d x .
\end{aligned}
$$

Set $R(\lambda)=R \lambda^{\infty / 2}$. By the strong Markov property and (3.13) and (3.15), for all $\lambda>0$ sufficiently small we have

$$
\begin{aligned}
& P^{x}\left[\int_{0}^{T_{0}} e^{-t} 1_{(0, R \wedge}{ }^{\alpha / 2)}(\phi(Z(t))) d t\right] \\
& \left.\quad=P^{s}\left\{e^{-T}{ }_{R(\lambda)} P^{Z(T}{ }_{R(\lambda)}\right)\left[\int_{0}^{T_{0}} e^{-t} 1_{\left(0, R \lambda^{\alpha / 2}\right)}(\phi(Z(t))) d \hat{\imath}\right]\right\} \\
& \quad \leqslant K e^{-\beta|x|} a R^{2 / \alpha} \lambda .
\end{aligned}
$$

Substituting this in (3.16) yields:

$$
\left|\int_{S} \lambda R_{\partial}^{\lambda} f(x) \rho(x) d x\right| \leqslant a K\|f\| R^{2 / \alpha} \lambda^{\alpha / 2} \int_{S} e^{-\beta|x|} \rho(x) d x .
$$

Since the right member above tends to zero as $\lambda \downarrow 0,(3.11)$ follows.

In principle, the nature of the excursions of $Z$ from $\{0\}$ is determined by $\left\{\eta^{\lambda}, \lambda>0\right\}$ and the killed semigroup $\left\{P_{t}^{\partial}, t>0\right\}$. However, it is not easy to deduce the path properties of the excursions from this representation. In particular, the answer to the following question is not obvious: "Does an excursion of $Z$ from $\{0\}$ stay in the interior of the wedge for a positive amount of time before reaching the boundary of the wedge or does it hit the boundary of the wedge immediately?" In the following theorem it is shown that the latter holds. An alternative phrasing of this result is that the excursions of $Z$ from $\{0\}$ do not share the local properties of the excursions from $\{0\}$ of Brownian motion conditioned to initially stay in the interior of the wedge.

Theorem 3.3. Let

$$
T_{\partial S}=\inf \{t>0: w(t) \in \partial S\} .
$$

Then

$$
\eta\left(T_{\partial S}>0\right)=0 \text {. }
$$

As a preliminary to the proof of this theorem, it is shown below that on certain sub- $\sigma$-algebras of $\mathscr{H}, \eta$ can be weakly approximated by the measures 
$P^{x}(\cdot) / \phi(x)$ as $|x| \downarrow 0$. Here $C_{S}$ is endowed with the Skorohod topology which is equivalent to the topology of uniform convergence on compact subsets of $[0, \infty)$.

For each $\varepsilon>0$ and $x \in S \backslash\{0\}$, let $P_{\varepsilon}^{x}$ be the sub-probability measure defined on $\left(C_{S}, \mathscr{M}\right)$ by

$$
P_{\varepsilon}^{x}(B)=P^{x}\left(w\left(\cdot \wedge T_{0}\right) \in B ; \sigma_{\varepsilon}<T_{0}\right), B \in \mathscr{M}
$$

where $\sigma_{\varepsilon}$ is given by

$$
\sigma_{\varepsilon}=\inf \{t \geqslant 0: \phi(w(t)) \geqslant \varepsilon\} .
$$

Lemma 3.4. For each $\varepsilon>0$, the family of measures

$$
\left\{P_{\varepsilon}^{x}(\cdot) / \phi(x): 0<\phi(x)<\varepsilon\right\}
$$

on $\left(C_{S}, \mathscr{M}\right)$ is bounded and tight.

Proof. Fix $\varepsilon>0$. For each $x \in S \backslash\{0\}$ such that $\phi(x)<\varepsilon$, by Corollary 2.1 of [16] we have

$$
P^{x}\left(\sigma_{\varepsilon}<T_{0}\right)=\phi(x) / \varepsilon
$$

and so each measure $P_{\varepsilon}^{x}(\cdot) / \phi(x)$ has total mass $\varepsilon^{-1}$. Define a modulus of continuity for $w \in C_{S}$ by

$$
\omega_{w}(\delta)=\sup \{|w(t)-w(s)|: 0 \leqslant s<t<\infty, t-s<\delta\}
$$

for each $\delta>0$. Then to prove the tightness, since the set of possible starting points for the family (3.20) is contained in a compact subset of $S$, it suffices to prove that for all $\beta>0$ sufficiently small,

$$
\lim _{\delta \downarrow 0} \lim _{|x| \rightarrow 0} \frac{P_{\varepsilon}^{x}\left(\omega_{w}(\delta)>\beta\right)}{\phi(x)}=0
$$

(cf. Billingsley [1, Theorem 8.2], Ethier-Kurtz [5, §3.7]). Now $\phi(x) \geqslant a|x|^{\alpha}$ where $a=\min _{\theta \in[0, \xi]} \cos \left(\alpha \theta-\theta_{1}\right)>0$ and so $\max \{|x|: \phi(x)=\beta\}=\left(\frac{\beta}{a}\right)^{1 / \alpha}$. Hence, for $0<\phi(x)<\beta<\varepsilon$,

$$
\begin{aligned}
& \frac{P_{\varepsilon}^{x}\left(\omega_{w}(\delta)>\beta+2\left(\frac{\beta}{a}\right)^{1 / \alpha}\right)}{\phi(x)} \leqslant \frac{P^{x}\left(\sigma_{\beta}<T_{0} ; P_{\varepsilon}^{w\left(\sigma_{\beta}\right)}\left(\omega_{w}(\delta)>\beta\right)\right)}{\phi(x)} \\
& \quad \leqslant \beta^{-1} \sup _{\phi(y)=\beta} P^{y}\left(\omega_{w}(\delta)>\beta\right) .
\end{aligned}
$$

To obtain the last inequality above, (3.21) has been used with $\beta$ in place of $\varepsilon$. 
Thus, to prove (3.22), it suffices to show the last expression above goes to zero as $\delta \downarrow 0$ for each fixed $\beta>0$. For this, by the strong Markov property of $\left\{P^{x}, x \in S\right\}$, it is enough to prove that

$$
\lim _{\delta \rightarrow 0} \sup _{x \in S} P^{x}\left(T_{x}(\beta) \leqslant \delta\right)=0
$$

where $T_{x}(\beta)$ is the first exit time of $w(\cdot)$ from a ball of radius $\beta$ centered at $x$ [5, §3.8]. Fix $\beta>0$ and choose $\gamma>0$ so small that $\gamma \leqslant \beta / 4$ and the ball of radius $\gamma$ centered at any $x \in\{z \in S:|z| \geqslant 3 \beta / 4\}$ intersects at most one side of S. Then [16, Theorem 2.4],

$$
\sup _{x \in S} P^{x}\left(T_{x}(\beta) \leqslant \delta\right) \leqslant \sup _{|x \geqslant| 3 \beta / 4} P^{x}\left(T_{x}(\gamma) \leqslant \delta\right)=o(1) \text { as } \delta \rightarrow 0
$$

and the tightness of (3.20) follows.

The next lemma characterizes any limit of (3.20) as $|x| \rightarrow 0$ on the sub$\sigma$-algebra $\mathscr{M}^{\varepsilon}$ of $\mathscr{M}$ generated by the sets of the form

$$
\left\{u^{\prime} \in C_{S}: w\left(t_{1}\right) \in A_{1}, \cdots, w\left(t_{k}\right) \in A_{k}\right\},
$$

where $A_{1}, \cdots, A_{k} \subset\{x \in S: \phi(x)>\varepsilon\}, 0<t_{1}<\cdots<t_{k}<\infty$ and $k$ ranges over the set of positive integers $\mathbb{N}$.

Lemma 3.5. Let $\varepsilon>0$ and $\mathcal{H}^{\varepsilon}$ be as described above. Suppose $Q_{\varepsilon}$ is a weak limit point of the family (3.20) as $|x| \rightarrow 0$. Then for each $B \in \mathcal{M}^{\varepsilon}$,

$$
Q_{\varepsilon}(B)=\chi\left(\psi_{1}\right) \eta(B)
$$

were $\chi\left(\psi_{1}\right)$ is defined by (1.14)-(1.15).

Remark. Because $\eta$ is a $\sigma$-finite measure and the characterization we have of $\eta$ is in terms of the Laplace transforms $\left\{\eta^{\lambda}, \lambda>0\right\}$, a more direct weak approximation to $\eta$ (e.g., $P^{x}\left(w^{\prime}\left(\cdot \wedge T_{0}\right) \in \cdot\right) / \phi(x)$ converges weakly to $\left.\eta(\cdot)\right)$ has not been obtained. However, Lemma 3.5 is sufficient for the purposes of this paper, since the sets $B_{\varepsilon \delta}$ defined in (3.29) below are in $\mathscr{M}^{\delta}$.

Proof. By the definition of $\mathscr{M}^{\varepsilon}$, it suffices to prove

$$
\begin{aligned}
& Q_{\varepsilon}\left(f_{1}\left(w \cdot\left(t_{1}\right)\right) f_{2}\left(w\left(t_{1}+t_{2}\right)\right) \cdots f_{k}\left(w\left(t_{1}+t_{k}\right)\right)\right) \\
& \quad=\chi\left(\psi_{1}\right) \eta\left(f_{1}\left(w\left(t_{1}\right)\right) f_{2}\left(w\left(t_{1}+t_{2}\right)\right) \cdots f_{k}\left(w\left(t_{1}+t_{k}\right)\right)\right)
\end{aligned}
$$

for $f_{1}, \cdots, f_{k}$ in $C_{c}^{\infty}(S)$ having support in $\{x \in S: \phi(x)>\varepsilon\}$ and $t_{1}>0,0<t_{2}<$ $\cdots<t_{k}<\infty, k \in \mathbb{N}$. The reason for singling out the time $t_{1}$ is that the proof uses the Markov property at the time $t_{1}$ and then the shifted times $t_{2}, \cdots, t_{k}$ appear (see (3.28) below). 
Suppose $\left\{x_{n}\right\}$ is a sequence in $S \backslash\{0\}$ such that as $n \rightarrow \infty,\left|x_{n}\right| \rightarrow 0$ and $\left\{P_{\varepsilon}^{x_{n}}(\cdot) / \phi\left(x_{n}\right)\right\}$ converges weakly to $Q_{\varepsilon}$. Then for $f_{1}, \cdots, f_{k}$ and $t_{1}, \cdots, t_{k}$ as described above,

$$
\begin{aligned}
\lim _{n \rightarrow \infty} & \frac{P_{\varepsilon}^{x_{n}}\left(f_{1}\left(w\left(t_{1}\right)\right) f_{2}\left(w\left(t_{1}+t_{2}\right)\right) \cdots f_{k}\left(w\left(t_{1}+t_{k}\right)\right)\right)}{\phi\left(x_{n}\right)} \\
= & Q_{\varepsilon}\left(f_{1}\left(w\left(t_{1}\right)\right) f_{2}\left(w\left(t_{1}+t_{2}\right)\right) \cdots f_{k}\left(w\left(t_{1}+t_{k}\right)\right)\right) .
\end{aligned}
$$

Since $P_{\varepsilon}^{x_{n}}(\cdot) / \phi\left(x_{n}\right)$ has total mass $\varepsilon^{-1}$ for all $n$, we have bounded convergence in (3.27). Moreover, using the fact that $f_{1}$ has support in $\{x \in S: \phi(x)>\varepsilon\}$ and the strong Markov property of $P^{x}$, we see that the limit in (3.27) may be rewritten as

$$
\lim _{n \rightarrow \infty} \frac{P^{x_{n}}\left(f\left(w\left(t_{1} \wedge T_{0}\right)\right)\right)}{\phi\left(x_{n}\right)}
$$

where $f \in C_{c}(S)$ is defined by

$$
f(x)=f_{1}(x) P^{x}\left(f_{2}\left(w\left(t_{2} \wedge T_{0}\right)\right) \cdots f_{k}\left(w\left(t_{k} \wedge T_{0}\right)\right)\right)
$$

and has support in $\{x \in S: \phi(x)>\varepsilon\}$. Thus, taking the Laplace transform of (3.27) in $t_{1}$, we obtain for each $\lambda>0$,

$$
\begin{aligned}
\lim _{n \rightarrow \infty} & \frac{R_{\partial}^{\lambda} f\left(x_{n}\right)}{\phi\left(x_{n}\right)}=\int_{0}^{\infty} e^{-\lambda t_{1}} \lim _{n \rightarrow \infty} \frac{P^{x_{n}}\left(f\left(w\left(t_{1} \wedge T_{0}\right)\right)\right)}{\phi\left(x_{n}\right)} d t_{1} \\
= & \int_{0}^{\infty} e^{-\lambda t_{1}} Q_{\varepsilon}\left(f_{1}\left(w\left(t_{1}\right)\right) f_{2}\left(w\left(t_{1}+t_{2}\right)\right) \cdots f_{k}\left(w\left(t_{1}+t_{k}\right)\right)\right) d t_{1} .
\end{aligned}
$$

But by (3.82) of [16], the limit on the left above is equal to $\chi\left(f-\lambda R_{\partial}^{\lambda} f\right)=$ $\chi\left(\psi_{1}\right) \eta^{\lambda}(f)$ (see equation (1.12)). Thus, by the continuity in $t_{1}$ of the integrands and uniqueness of the Laplace transform, it follows that $\chi\left(\psi_{1}\right) \eta_{t_{1}}(f)$ is equal to the right member of (3.27) for all $t_{1}>0$. But, by the Markov property of $\eta(3.6)$ and $P_{t}^{\partial}$, this is equivalent to (3.26).

Proof of Theorem 3.3.

For each $\varepsilon \in(0,1)$ and $0<\delta<\varepsilon$, let $\sigma_{\varepsilon}$ be defined by (3.19) and $T_{\delta}$ be defined by (3.14) with $r=\delta$ and define

$$
T_{\delta}^{\varepsilon}=\inf \left\{t \geqslant \sigma_{\varepsilon}: \phi(w(t)) \leqslant \delta\right\} \equiv \sigma_{\varepsilon}+T_{\delta} \circ \theta_{\sigma_{\varepsilon}} .
$$

Now, $\eta$-a.s., $\sigma_{\varepsilon}$ is a left continuous, increasing function of $\varepsilon \in(0, \infty)$ and so the set of points of discontinuity of $\varepsilon \rightarrow \sigma_{\varepsilon}$ is at most countable. Hence by Fubini's theorem, for almost every $\varepsilon>0$,

$$
\eta\left(\lim _{\beta \rightarrow \varepsilon_{+}} \sigma_{\beta} \neq \sigma_{\varepsilon} \text { or } \lim _{\beta \rightarrow \varepsilon-} \sigma_{\beta} \neq \sigma_{\varepsilon}\right)=0 .
$$


Thus, we can choose a sequence of $\varepsilon^{\prime}$ s converging monotonically to zero such that $\sigma_{\varepsilon}$ is $\eta$-a.s. a continuous functional on $C_{S}$ (cf. Kurtz [9, pp. 13-14]). Fix an $\varepsilon>0$ in such a sequence and let $t>0$. Since $\eta$-a.s., $T_{\delta}^{\varepsilon}$ is right continuous and decreasing as a function of $\delta$, by similar reasoning to that above, there is a sequence of $\delta^{\prime}$ s (possibly depending on $\varepsilon$ ) tending monotonically to zero for which $T_{\delta}^{\varepsilon}$ is $\eta$-a.s. a continuous functional on $C_{S}$. Then, by the continuity properties of $\sigma_{\varepsilon}, \sigma_{\varepsilon}+t$ and $T_{\delta}^{\varepsilon}$ on $C_{S}$, and of

$$
h h_{f}^{ \pm}(r, u, w)=\left(\begin{array}{c}
\min \\
\max
\end{array}\right)\{f(w(s)): r \leqslant s \leqslant u\}
$$

on $[0, \infty) \times[0, \infty) \times C_{S}$ when $f=\phi \wedge 1$ or $f$ is a continuous function on $S$ equal to $\arg (\cdot)$ (the polar angle) on $\{x \in S: \phi(x) \geqslant \delta\}$, it follows that $B_{\mathrm{z} \delta}$, defined below, is an open subset of $C_{S}$.

$$
\begin{aligned}
B_{\varepsilon \delta}= & \left\{\sigma_{\varepsilon}<\infty,\left(T_{\partial s} \wedge T_{\delta}\right) \circ \theta_{\sigma_{\varepsilon}}>t\right\} \\
= & \left\{\sigma_{\varepsilon}<\infty, \min _{\sigma_{\varepsilon} \leqslant s \leqslant \sigma_{\varepsilon}+t} \phi(w(s))>\delta,\right. \\
& \min \left(\arg (w(s)): \sigma_{\varepsilon} \leqslant s \leqslant\left(\sigma_{\varepsilon}+t\right) \wedge T_{\delta}^{\varepsilon}\right)>0, \\
& \left.\max \left(\arg (w(s)): \sigma_{\varepsilon} \leqslant s \leqslant\left(\sigma_{\varepsilon}+t\right) \wedge T_{\delta}^{\varepsilon}\right)<\xi\right\} .
\end{aligned}
$$

The set $B_{\varepsilon \delta}$ is in $\mathscr{H}^{\delta}$ and so by Lemma 3.5 , for any weak limit point $Q_{\delta}$ of $P_{\delta}^{x}(\cdot) / \phi(x)$ as $|x| \rightarrow 0$, we have

$$
Q_{\delta}\left(B_{\varepsilon \delta}\right)=c_{1} \eta\left(B_{\varepsilon \delta}\right)
$$

where $c_{1}=\chi\left(\psi_{1}\right)$. Then letting $\lim _{\delta \psi^{\circ}}$ denote the limit along the sequence of $\delta^{\prime} \mathrm{s}$ chosen above, we obtain

$$
\eta\left(T_{\partial S}>\sigma_{\varepsilon}+t\right)=\lim _{\delta \downarrow 0} \eta\left(B_{\varepsilon \delta}\right)=\lim _{\delta \downarrow 0} c_{1}^{-1} Q_{\delta}\left(B_{\varepsilon \delta}\right) .
$$

Since $B_{\varepsilon \delta}$ is open in $C_{S}$ [5, Theorem 3.1, p. 108],

$$
Q_{\delta}\left(B_{\varepsilon \delta}, \leqslant \liminf _{x \rightarrow 0} \frac{P_{\delta}^{x}\left(B_{\varepsilon \delta}\right)}{\phi(x)},\right.
$$

where the limit as $x$ tends to zero is along any sequence of $x$ 's such that $P_{\delta}^{x}(\cdot) / \phi(x)$ converges weakly to $Q_{\delta}$. By the definitions of $P_{\delta}^{x}$ and $B_{\varepsilon \delta}$, and the strong Markov property of $P^{x}$, the right member of (3.31) is equal to

$$
\begin{gathered}
\liminf _{x \rightarrow 0} \frac{P^{x}\left(\sigma_{\varepsilon}<T_{0} ; P^{w\left(\sigma_{\varepsilon}\right)}\left(T_{\partial S} \wedge T_{\delta}>t\right)\right)}{\phi(x)}- \\
\quad \leqslant \liminf _{x \rightarrow 0} \frac{P^{x}\left(\sigma_{\varepsilon}<T_{0}\right)}{\phi(x)} \sup _{\phi(y)=\varepsilon} P^{y}\left(T_{\partial S}>t\right) .
\end{gathered}
$$

In [13], Shimura obtained asymptotic properties of the transition probabilities 
for Brownian motion in a wedge with absorption at the boundary. In particular, formula (4.2) of his paper yields the following estimate. There is a constant $c_{2}>0$, depending on $t$, but not on $\varepsilon>0$, such that

$$
\sup _{\phi(y)=\varepsilon} P^{y}\left(T_{\partial S}>t\right) \leqslant c_{2} \varepsilon^{\nu / \alpha}
$$

where $\nu=\frac{\pi}{\xi}$ and so $\frac{\nu}{\alpha}=\frac{\pi}{\theta_{1}+\theta_{2}}$. The fact that $\phi(y) \sim|y|^{\alpha}$ has been used here. Substituting (3.33) and (3.21) in (3.32) yields

$$
\eta\left(T_{\partial S}>\sigma_{\varepsilon}+t\right) \leqslant c_{3} \varepsilon^{\left(\pi-\theta_{1}-\theta_{2}\right) /\left(\theta_{1}+\theta_{2}\right)}
$$

where $c_{3}=c_{1}^{-1} c_{2}$ and $\theta_{1}+\theta_{2}<\pi$. Letting $\varepsilon \downarrow 0$ yields

$$
\eta\left(T_{\partial S}>t\right)=0 \text {. }
$$

Since $t>0$ was arbitrary, the desired result (3.18) follows.

\section{Acknowledgement}

The author would like to thank Professor K. Itô and Peter March for stimulating discussions on this work and J.-F. Le Gall for conversations on this and related research.

\section{References}

[1] Billingsley, P., Cpnvergence of Probability Measures, John Wiley and Sons, New York, 1968.

[2] Blumenthal, R.M., and Getoor, R.K., A dimension theorem for sample functions of stable processes, Ill. J. Math., 5 (1960), 370-375.

[3] —-, Markov Processes and Potential Theory, Academic Press, 1968.

[4] Cinlar, E., Jacod, J., Protter, P., and Sharpe, M.J., Semimartingales and Markov processes, Z. Wahr. verw. Geb., 54 (1980), 161-219.

[5] Ethier, S.N., and Kurtz, T.G., Markov Processes-Characterization and Convergence, John Wiley and Sons, New York, 1986.

[ 6 ] Getoor, R.K., Excursions of a Markov process, Annals Probability, 7 (1979), 244-266.

[7] Itô, K., Poisson puint processes attached to Markov processes, Proc. Sixth Berkeley Symposium, Vol. III, Univ. of California Press 1972, 225-239.

[8] Jacod, J., Calcul Stochastique et Problemes de Martingales, Lecture Notes in Math. No. 714, Springer, New York, 1979.

[9] Kurtz, T.G., Approximation of population processes, CBMS-NSF Regional Conference Series in Applied Mathematics, SIAM, Philadelphia, 1981.

[10] Le Gall, J.-F., Mouvement brownien, cônes et processus stables, preprint, 1986

[11] Maisonneuve, B., Exit systems, Annals Probatility, 3 (1975), 399-411.

[12] Salisbury, T.S., On the Itô excursion process, to appear in Probability Theory and Related Fields.

[13] Shimura, M., Excursions in a cone for two-dimensional Brownian motion, J. Math 
Kyoto Univ., 25 (1985), 433-443.

[14] Taylor, S.J., Sample path properties of processes with stationary independent increments, in Stochastic Analysis, ed. D.G. Kendall and E.F. Harding, John Wiley and Sons, 1973, 387-414.

[15] Taylor, S.J., and Wendel, J.G., The exact Hausdorff measure of the zero set of a stable process, Z. Wahr. verw. Geb., 6 (1966), 170-180.

[16] Varadhan, S.R.S., and Williams, R.J., Brownian motion in a wedge with oblique reflection, Comm. Pure Appl. Math., 38 (1985), 405-443.

[17] Williams, R.J., Recurrence classification and invariant measure for refiected Brownian motion in a wedge, Annals Probability, 13 (1985), 758-778.

[18] —-, Reflected Brownian motion with skew symmetric data in a polyhedral domain, to appear in Probability Theory and Related Fields. 
